\section{Beyond 3D Building Modelling: Citizen Science for 3D Cultural Mapping}

Gl_Forum 2019, Issue 2

Page: 3 - 9

Short Paper

Corresponding Author:

gisszymon@gmail.com

DOI: 10.1553/giscience2019_02_s3

\author{
Szymon Chmielewski', Cait Bailey² and Adam Gawryluk \\ IUniversity of Life Sciences in Lublin, Poland \\ ${ }^{2} \mathrm{MDI}$ Biological Laboratory, Maine, USA
}

\begin{abstract}
This paper discusses a research-in-progress project engaging citizens in the collaborative 3D reconstruction of special urban features (SUFs) - visual works of art located in urban public spaces. While most artists modelling urban public spaces use basic 3D building models, these models do not include visual artworks. Because urban planners use artists' models when designing urban public spaces, we believe it is necessary to incorporate SUFs into 3D city models to help urban planners and artists visualize urban spaces more effectively. To achieve this goal, we propose a bottom-up approach to the geographic visualization of SUF cultural assets (i.e. cultural mapping) which so far has not been undertaken as a citizen science project. The main aims of this project are to engage citizens in contributing to 3D SUF models and to familiarize them with citizen science. The method includes crowdsourcing SUF data and analysing it using 'structure from motion' (SfM) algorithms. After a 6-month campaign in Lublin, Poland, this project has so far resulted in 271 observations and 144 collaborative 3D models, creating a 3D open-data product which can be used for further culturally sensitive urban planning purposes.
\end{abstract}

\title{
Keywords:
}

cultural mapping, citizen science, 3D city models, Anecdata

\section{Introduction}

3D city models provide a sophisticated virtual environment for spatial analysis, both in professional 3D GIS software and on the Web. These models serve as communication tools between urban planners (Ranzinger \& Gleixner 1997; Koziatek \& Dragićević, 2017) and policymakers (Batty et al., 2000), while also helping to improve public participation in sustainable urban planning processes (Wanarat \& Nuanwan, 2013). 3D city models typically consist of three basic layers: elevation models, building models and vegetation models. Buildings are the most dominant feature of the urban environment and serve as the most significant feature in 3D city models (Biljecki, 2017). Smaller and more complex objects are often missed, despite their importance to the visual characteristics of a city. Because of their profound aesthetic and artistic qualities, these are classified as Special Urban Features (SUFs) (Crowhurst \& Henry, 1987), and our preliminary study is an attempt to incorporate SUFs in 
3D city models. SUF typically refers to public visual art (Nasar, 1989), or artworks created with the participation of community members and selected artists, which are located in urban public spaces (Jasmi, \& Mohamad, 2016). Because urban planners use artists' models when designing urban public spaces, we found it advisable to incorporate SUF models into 3D city models in order to extend urban planners' and artists' abilities to visualize urban spaces, and to extend the capability of 3D city models to help implement a culturally sensitive urban policy (Kagan et al., 2018; Redaelli, 2012).

Cultural mapping, or the process of collecting and analysing data on cultural assets, including SUFs (Cauchi-Santoro, 2016), is a key aspect of public participation in cultural planning (Duxbury 2015, Redaelli, 2012). As a bottom-up process engaging local communities to gather and analyse data on cultural assets (Redaelli, 2015; Duxbury, 2015), cultural mapping can be viewed as a citizen science (CS) task. The aim of this project is to engage citizens in contributing to 3D SUF models and to familiarize them with CS. This study answers the question of how successful cultural mapping can be undertaken as a CS project. It was carried out in the city of Lublin in eastern Poland, which was a contestant to become the European capital of culture in 2016.

The potential of CS, understood as public participation in scientific research (Bonney et al., 2009), arises from the participation of volunteers (Haklay, 2018). The fuzzy boundaries of CS definitions and applications (Haklay, 2013) also allow for its extension to include opportunities for learning science. This research-in-progress paper discusses the use of a free CS geoweb platform (Kar et al., 2016) to host a 3D cultural mapping project. The proposed framework enables the collaborative 3D reconstruction of SUFs, where participants not only collect observations but also analyse them using structure from motion ( $\mathrm{SfM}$ ) software. Moreover, as an academic project, it also aims to turn students into citizen scientists (Harlin et al. 2018).

\section{Development method}

The method used in the '3D visual art project' fits into the general CS workflow (Bonney et al., 2009). However, the project's multiple strands and interdisciplinary nature call for special considerations in the framework's design. The three-step method consists of (1) SUF photo crowdsourcing, (2) 3D reconstruction, and (3) data fusion and WebScene publication (Figure 1). 


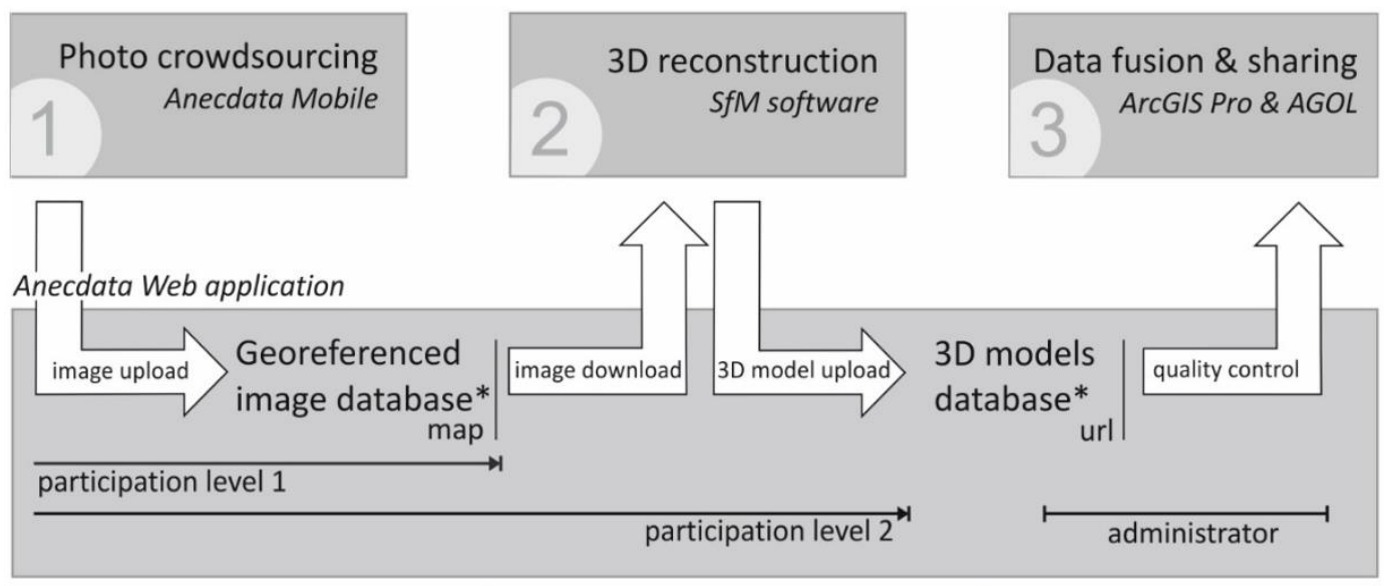

*elements can be commented on or flagged by the project members to improve data quality

Figure 1: Flowchart of the method along with citizens' engagement levels: level 1 - photo crowdsourcing only; level 2 - uploaded image analysis and 3D model reconstruction.

\subsection{Crowdsourcing geotagged photos}

The project uses existing CS geoweb infrastructure to collect and share observations. Because it requires uploading and downloading high-resolution images, we decided to use Anecdata.org, an online citizen science platform used by a wide range of research projects across the world. In the context of this study, citizens use the Anecdata.org mobile app to capture multiple geo-referenced photographs of SUFs. The photographs are then immediately available for viewing, comment and download.

\subsection{Reconstruction of 3D models and WebScene presentation}

Of the various 3D modelling methods (scanning, photogrammetry, CAD-based and rulebased), we decided to use SfM 3D reconstruction due to its availability to any citizen who has the capacity to take photos. Furthermore, the proposed wizard-based SfM software is simple enough for use by citizen scientists. Our workflow recommends using Zephyr 3DF, but volunteers are free to choose another package as long as it supports the Collada or OBJ format. Zephyr 3DF is a turn-key solution which automatically analyses objects in images to infer the orientation of the camera. Once it determines the angles and distances from which each image was captured, it generates a 3D model mesh and stitches the images together to create a model texture. The user then shares their completed SUF model via the URL. To train volunteers in 3D reconstruction, workshops are held periodically at the University of Life Sciences in Lublin as well as at the local 'Warsztaty Kultury' media lab.

\subsection{Data fusion and WebScene publication}

Completed submissions on the Anecdata.org platform consist of a URL linking to the usergenerated model, an image preview of the 3D model, as well as all the original photographs which were used for 3D reconstruction. Members can comment on user-generated models if 
they appear to have errors, or to share links to other 3D models proposed by other members. Low-quality models and content not suitable for SfM can be marked by project participants for administrator review. Project administrators choose models for publication and update the WebScene periodically using ArcGIS Online.

\section{Results}

\subsection{The crowdsourcing campaign results}

The project started in the middle of October 2018 as part of the GIS Day event at the University of Life Sciences in Lublin. 59 participants registered, of whom 35 (59.3\%) said they were willing to collect photos. Because of University computer lab's time limitations, only 28 people were eventually invited to participate, received technical instruction, and were briefed on the project's goals. This initial photo collection campaign was carried out over 4 days (1013 October), and 1,734 photos were uploaded. The most active volunteer was '@bk-Marlena', who uploaded 490 photos over the course of the campaign. A total of 55 visual art objects were photographed, including 30 sculptures, 4 installations, 3 building façades, and 18 small architectural objects. So far (June 2019), the project has 87 members, of whom 69 are students and 18 are Lublin residents recruited at a 'Warsztaty Kultury' media lab workshop on 25 April. In total, 432 observations have been submitted, of which 391 are located in Lublin. Over half of the observations recorded in Lublin (69.3\%) meet the requirement of having the 10 or more photos to make them useful for further 3D reconstruction. Currently, the project hosts 144 links to 3D models which are used for further WebScene publication.

The project webpage (https://www.anecdata.org/projects/view/442) provides a 2D map of observations where project progress can be tracked. So far, the most recognizable visual art object in the Lublin case study is the 'Hand' sculpture by Paweł Pawluk (Figure 2).

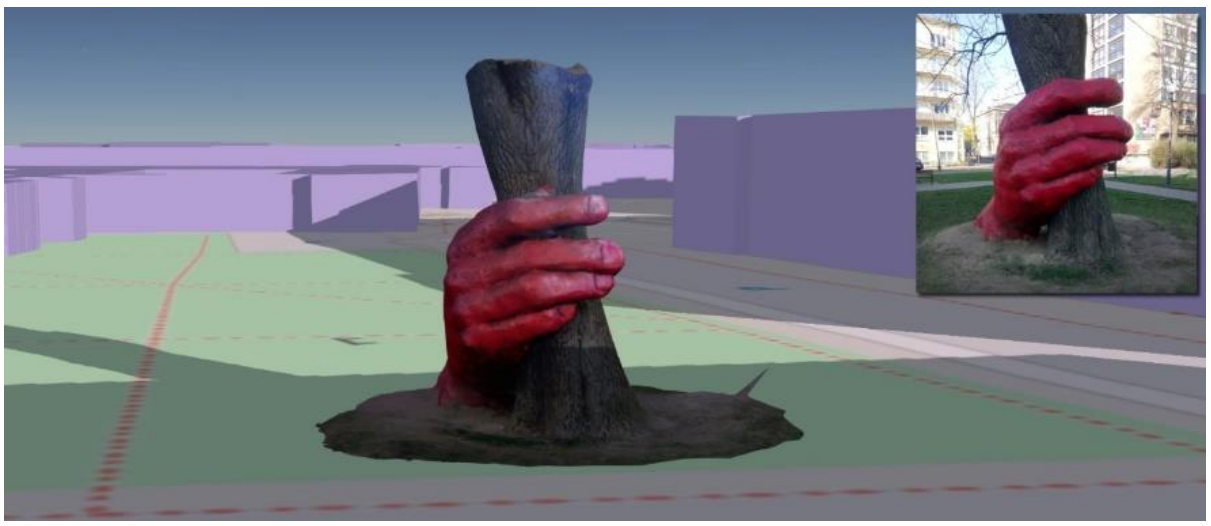

Figure 2: 'Hand': original image (top right) and 3D WebScence (main image). 


\subsection{The results of Geoweb platform parameter adjustment}

The fruitful collaboration with Anecdata.org has resulted in technical improvements to the geoweb platform. Since January 2019, all photos are stored and shared in high resolution (3,200 x 2,400 pixels) and up to 50 images per single observation upload. The Anecdata team updated their online image galleries to provide improved image previews, as well as the ability to reorder images so that reconstructed 3D models appear first. 'Visual Art 3D' users' photos are annotated with the following attributes: city, GPS status, visual art category, and an optional description. Basic statistics on the project members and the number of their observations are available. Both the Web and the mobile versions of Anecdata provide a link to 3D City WebScene, which allows a virtual tour through reconstructed SUFs.

\subsection{WebScene visualization}

The scene uses a default digital elevation model with customizable basemaps; the building models are represented at LoD1 using subdued colour. The panel on the right allows the viewer to switch layers and conduct shadow analysis. The user can navigate between reconstructed models via bookmarks at the bottom of the scene view (Figure 3), or by zooming to the selected layer. The scene is shared as a link (https://arcg.is/0zPCPD) and can be viewed in any web browser.

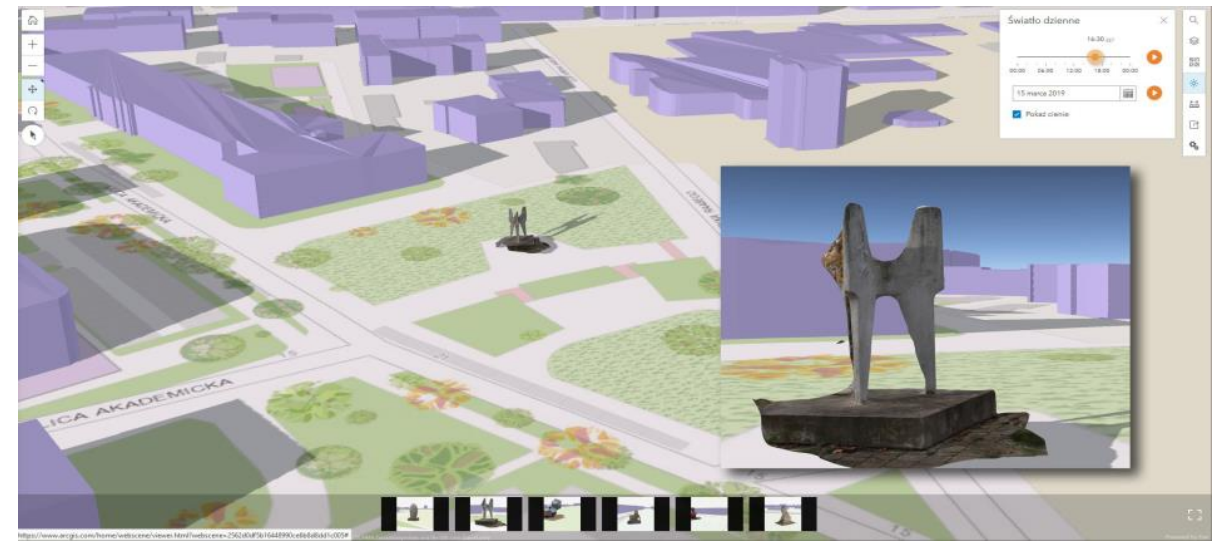

Figure 3: The Raabego sculpture - SUF visualization with shadow casting.

\section{Conclusions}

In this study, we apply citizen science to the field of cultural mapping by integrating SUFs with 3D city models to help urban planners and artists visualize urban space more effectively. This is not a trivial task. From a technical point of view, the image collection, 3D reconstruction and visualization could certainly be implemented as a Platform As A Service (PAAS) cloud service (see e.g. Tefera et al., 2018), which would streamline user experience and reduce the number of software products used in this case study. Other similar, non-expert but citizen- 
driven case studies of producing image-based 3D models (Rahaman, Champion \& Bekele, 2019) confirm the demand for SfM-ready CS platforms. This study, by using Anecdata, overcomes the basic technical limitations of most CS geoweb platforms: most limit the resolution of uploaded images and file size. For example, Epicollect5 allows unlimited images, but the resolution is reduced to 1,024 pixels and images are highly compressed. The CitSci and GeoODK mobile platforms do not compress images as much, but they impose a limit on file size. Unfortunately, this reasonable policy makes them unsuitable for storing photos for 3D reconstruction. In conclusion, the technical limitations of geoweb platforms makes neophotogrammetry (Leberl, 2010) difficult to use in citizen science.

The study results also yield social conclusions regarding citizens' engagement. If crowdsourcing is defined here as data collection and CS is data collection and analysis, then this project makes both activities possible and allows participants to adjust their involvement according to their skills levels. Tasks that may seem simple to professionals, such as taking photos for SfM reconstruction, may prove difficult for the general public. Providing hands-on interactive training for teachers and engaging students and volunteers in workshops (Harlin et al., 2018) allows them to teach each other how to reconstruct and visualize the city's cultural assets and thereby become citizen scientists.

\section{Acknowledgements}

Mobile app development was funded with a Maine Technology Institute Seed Grant \#SG5554 and funding from the Alex C. Walker Foundation. We also thank Agnieszka Wojciechowska and Ewa Orzeszko from medialab 'Warsztaty Kultury' for organizing two citizen science workshops for Lublin city-dwellers.

\section{References:}

Batty, M., David, C., Steve, E., Mordechai, H., Stefan, K., Naru, S., et al. (2000). Visualizing the City: Communicating Urban Design to Planners and Decision Makers. London: CASA, UCL Working Paper 26.

Biljecki, F. (2017). Level of detail in 3D city models. PhD thesis, TU Delft, 353 pp.

Bonney, R., Cooper, C. B., Dickinson, J., Kelling, S., Phillips, T., Rosenberg, K. V. and Shirk, J. (2009). Citizen science: A developing tool for expanding science knowledge and scientific literacy. BioScience, 59(11). 977-84. DOI:10.1525/ bio.2009.59.11.9

Cauchi-Santoro, R. (2016). Mapping community identity: Safeguarding the memories of a city's downtown core. City, Culture and Society, 7(1), 43-54

Crowhurst, L., Henry, L. (1987). Livable cities - People and places: Social and design principles for the future of the city. Southampton and New York: Gondolier Press.

Duxbury, N. (2015). Cultural Mapping as Cultural Inquiry: Introduction to an Emerging Field of Practice. Duxbury N., Garrett-Petts, W.F., MacLennan, D. eds. Routledge Advances in Research Methods: Cultural Mapping as Cultural Inquiry. Florence, KY: Taylor and Francis.

Haklay, M. (2013). Citizen Science and Volunteered Geographic Information - overview and typology of participation in Sui, D.Z., Elwood, S. and M.F. Goodchild (eds.), Crowdsourcing Geographic 
Knowledge: Volunteered Geographic Information (VGI) in Theory and Practice. Berlin: Springer. pp 105-122 DOI: 10.1007/978-94-007-4587-2_7

Haklay, M. (2018). Participatory citizen science. In: Hecker, S., Haklay, M., Bowser, A., Makuch, Z., Vogel, J. \& Bonn, A. Citizen Science: Innovation in Open Science, Society and Policy. UCL Press, London. (pp. 52-62).

Harlin, J., Kloetzer, L., Patton, D., Leonhard, Ch. (2018). Turning students into citizen scientists. in: Hecker, S., Haklay, M., Bowser, A., Makuch, Z., Vogel, J. \& Bonn, A. Citizen Science: Innovation in Open Science, Society and Policy. UCL Press, London

Jasmi, M. \& Mohamad, N. (2016). Roles of Public Art in Malaysian Urban Landscape towards Improving Quality of Life: Between Aesthetic and Functional Value. Procedia - Social and Behavioral Sciences, 222, 872-880.

Kagan, S., Hauerwaas, A., Holz, V., \& Wedler, P. (2018). Culture in sustainable urban development: Practices and policies for spaces of possibility and institutional innovations. City, Culture and Society, 13, 32-45. https://doi.org/10.1016/j.ccs.2017.09.005

Kar, B., Sieber, R., Haklay, M., \& Ghose, R. (2016). Public Participation GIS and Participatory GIS in the Era of GeoWeb. Cartographic Journal. https://doi.org/10.1080/00087041.2016.1256963

Koziatek, O., \& Dragićević, S. (2017). iCity 3D: A geosimualtion method and tool for three-dimensional modeling of vertical urban development. Landscape and Urban Planning, 167, 356367. https://doi.org/10.1016/j.landurbplan.2017.06.021

Leberl, F. (2010). Time for Neo-Photogrammetry. GIS Development, 14. 22-24.

Nasar, J.L. (1989). Perception, cognition and evaluation of urban places. In Altman, I \& Zube E.H. (Eds). Public Places and Spaces. New York and London: Plenum Press

Rahaman, H., Champion, E., \& Bekele, M. (2019). From photo to 3D to mixed reality: A complete workflow for cultural heritage visualisation and experience. Digital Applications in Archaeology and Cultural Heritage, 13. https://doi.org/10.1016/j.daach.2019.e00102

Ranzinger, M., \& Gleixner, G. (1997). GIS datasets for 3D urban planning. Computers, Environment and Urban Systems, 21(2), 159-173. https://doi.org/10.1016/S0198-9715(97)10005-9

Redaelli, E. (2012). Cultural Planning in the United States: Toward Authentic Participation Using GIS. Urban Affairs Review, 48(5), 642-669. https://doi.org/10.1177/1078087412441158

Redaelli, E. (2015). Cultural mapping - analysing its meanings in policy making. In: Duxbury, N., Garrett-Petts, W.F., MacLennan, D., (eds.) Cultural Mapping as Cultural Inquiry. Routledge Advances in Research Methods, Florence, KY: Taylor and Francis, pp. 86-98.

Tefera, Y., Poiesi, F., Morabito, D., Remondino, F., Nocerino, E., \& Chippendale, P. (2018). 3Dnow: Image-Based 3D Reconstruction and Modeling Via Web. ISPRS - International Archives of the Photogrammetry, Remote Sensing and Spatial Information Sciences, XLII 2, 1097-1103. https://doi.org/10.5194/isprs-archives-XLII-2-1097-2018

Wanarat, K., \& Nuanwan, T. (2013). Using 3D Visualisation to Improve Public Participation in Sustainable Planning Process: Experiences through the Creation of Koh Mudsum Plan, Thailand. Procedia - Social and Behavioral Sciences, 91, 679-690. https://doi.org/10.1016/j.sbspro.2013.08.469 